\title{
SARS-CoV-2 viral shedding among diabetic patients: from upper to lower respiratory tract
}

\author{
Pierpaolo Trimboli $\mathbb{C}^{1,2} \cdot$ Enos Bernasconi ${ }^{2,3} \cdot$ Niccolo Buetti $^{4,5,6}$
}

Received: 22 October 2020 / Accepted: 11 November 2020 / Published online: 22 November 2020

(c) Springer Science+Business Media, LLC, part of Springer Nature 2020

To the Editor,

We would like to thank Cano et al. for their interest in our manuscript "Diabetes mellitus is a risk factor for prolonged SARS-CoV-2 viral shedding in lower respiratory tract samples of critically ill patients" [1]. Data on SARSCoV-2 viral shedding in COVID-19 patients are conflicting and this topic achieves high interest. The recently published letter by Cano et al. suggested that patients with diabetes mellitus (DM) did not show prolonged RNA viral shedding based on nasopharyngeal sampling [2]. At first glance, these data seem to be in contrast to our study showing a prolonged viral shedding in lower respiratory tract of critically ill patients [1]. However, we respectfully would underline that this discrepancy could depend on differences in demographic feature and methodological/ statistical approaches. Then, the following two issues have to be considered when comparing our results [1] with that from Cano et al. [2]:

(1) Viral shedding in critically ill patients remains unexplored. Cano et al. in their original publication, mostly analyzed outpatients (189/251), being only $25 \%$ of these patients were hospitalized [3]. We, therefore, suppose that critically ill patients were only a minority of these patients. Critically ill patients represent a population severely affected by SARS$\mathrm{CoV}-2$ that is not comparable with outpatients. Moreover, all our patients had an acute respiratory

Pierpaolo Trimboli

pierpaolo.trimboli@eoc.ch

1 Clinic for Endocrinology and Diabetology, Lugano Regional Hospital, Ente Ospedaliero Cantonale, Lugano, Switzerland

2 Faculty of Biomedical Sciences, Università della Svizzera Italiana (USI), Lugano, Switzerland

3 Ente Ospedaliero Cantonale, Division of Infectious Diseases, Regional Hospital Lugano, Lugano, Switzerland distress syndrome requiring invasive mechanical ventilation, which may influence the viral presence in the respiratory tract.

(2) Several methodological differences between our analysis and the Cano's study should be highlighted. We performed a biweekly screening on lower respiratory tract samples in all patients, which is probably a different sample collection strategy compared to the patients included by Cano and colleagues. In addition, to evaluate risk factors for prolonged viral shedding, we used the survival Cox models [1], which are different to univariate regression models used by Cano et al. [2]. Moreover, both studies included a limited number of patients and the statistical analyses should be interpreted with caution: It is conceivable that a larger samples size would allow to define more correctly risk factors for prolonged viral shedding. Finally, polymerase-chain reaction SARS-CoV-2 tests used in both studies may overestimate the true duration of viral shedding (i.e., assessed by viral cell cultures).

We advise for further investigations about the relationship between DM and SARS-CoV-2 infection.

Acknowledgements We thank the Swiss National Science foundation, the Bangerter-Rhyner Foundation and the Ente Ospedaliero Cantonale (regional public hospitals network, EOC).

4 Ente Ospedaliero Cantonale, Regional Hospital Locarno, Locarno, Switzerland

5 University of Paris, INSERM IAME, U1137, Team DeSCID, Paris, France

6 Infection Control Program and World Health Organization Collaborating Centre on Patient Safety, University Hospitals and Faculty of Medicine, University of Geneva, Geneva, Switzerland 
Funding NB is currently receiving a Post.doc Mobility grant from the Swiss National Science Foundation (grant number: P400PM_183865) and a grant from the Bangerter-Rhyner Foundation. These grants support his fellowship in Paris. The Ente Ospedaliero Cantonale (regional public hospitals network, EOC) supported this work.

Author contributions All authors contributed to the letter conception and design. The first draft of the manuscript was written by NB and PT; and EB commented on previous versions of the manuscript. All authors read and approved the final manuscript.

\section{Compliance with ethical standards}

Conflict of interest The authors declare that they have no conflict of interest.

Ethical approval This article does not contain any studies with human participants or animals performed by any of the authors.
Publisher's note Springer Nature remains neutral with regard to jurisdictional claims in published maps and institutional affiliations.

\section{References}

1. N. Buetti, P. Trimboli, T. Mazzuchelli, E. Lo Priore, C. Balmelli, A. Trkola, M. Conti, G. Martinetti, L. Elzi, A. Ceschi., V. Consonni, A. Ogna, V. Forni-Ogna, E. Bernasconi, Diabetes mellitus is a risk factor for prolonged SARS-CoV-2 viral shedding in lower respiratory tract samples of critically ill patients. Endocrine (2020). https://doi.org/10.1007/s12020-020-02465-4

2. E. Cano, C. Corsini Campioli, J.C O'Horo, Nasopharyngeal SARSCoV-2 viral RNA shedding in patients with diabetes mellitus. Endocrine (2020). https://doi.org/10.1007/s12020-020-02516-w

3. C. Corsini Campioli, E. Cano Cevallos, M. Assi, R. Patel, M.J. Binnicker, J.C. O'Horo, Clinical predictors and timing of cessation of viral RNA shedding in patients with COVID-19. J. Clin. Virol. (2020). https://doi.org/10.1016/j.jcv.2020.104577 